\title{
Entomofauna investigations in the apricot orchards, Prunus ar- meniaca L. (Rosales Rosaceae), in Ouled Si Slimane, Batna, North East Algeria
}

\author{
Smail Chafaa ${ }^{*}$, Sabrina Belkhadria' \& Fateh Mimeche ${ }^{2}$ \\ ${ }^{1}$ Department of Ecology and Environment, University of Batna 2, 05078 Batna, Algeria \\ ${ }^{2}$ Department of Agricultural Sciences, University of M'Sila, 28000 M'Sila, Algeria \\ "Corresponding author, e-mail: chafaasmail@gmail.com
}

ABSTRACT

Researches carried out in the past years allowed to list an entomofauna that lives on the apricot orchards, Prunus armeniaca L. (Rosales Rosaceae), in Ouled Si Slimane (Batna Department, Algeria). The investigated period was from October 2014 until May 2015. The sampling techniques used were: hunting in sight, barber pots, colorful traps, entomological umbrella and the filleting net. We have identified a total of 125 species divided into 9 orders and 54 families in which Coleoptera and Hymenoptera were quantitatively represented. The phytophagous were the most present. Its Shannon Value exceeds 4.5 Bits and indicates a Specific Richness of the insects and diversification of ecological niches in this kind of fauna.

\section{INTRODUCTION}

In Algeria, apricot cultivation, Prunus armeniaca L. (Rosales Rosaceae), is distributed in coastal plains, Tellian Atlas, high plateaus, pre-desert areas and Saharan Oases (Chafaa, 1992). The apricot's pests are few and rarely specific. Their population varies significantly in space and time (Chafaa, 1992). According to Vidaud (1980), these pests are generally classified according to their level of attack. The root, neck, trunk and branch pests are represented by several species (Hariri, 1978; Bretaudeau, 1979; Malek, 1987).

Due to the economic and social importance of apricot, its culture has been the subject of research and experimentation for years to improve its productivity.

In Algeria, some works are published on the apricot: Chaouia (1987), on the varietal behaviour of the species in the Boufarik experimental station (Blida Department), Bouzidi (1990) in Batna Department, Derias (1984), Benabbes (1990) and Achourri (1991) in the region of Ain Touta and on apricot berry dieback in the N'Gaous region, Chafaa (1992) in the region of Ouled Si Slimane on the ecological biology of apricot zoocoenosis. Numerous are the publications on this subject also in other countries (see, for example: Ivanova, 1991, 2006; Dér et al., 2003; Bonsignore et al., 2008).

This work has the main objective to highlight apricot entomofauna subservient to the agro-systems in Ouled Si Slimane orchards. Thus, we aim in our study to know the bio-ecological status of the different insect groups: abundance, diversity and diet. 


\section{MATERIAL AND METHODS}

Our study was conducted in an apricot orchard in the Kochbi region located about $5 \mathrm{~km}$ from Ouled Si Slimane city (Lat. 35³6’39'N, Long. $\left.5^{\circ} 37^{\prime} 58^{\prime \prime} \mathrm{E}\right)$. This region is $90 \mathrm{~km}$ from Batna department, in north east Algeria. The climate of the study area during the period 1985-2014 was characterized by a total average precipitation of 251.59 $\mathrm{mm}$, the average temperature varied between $0.03^{\circ} \mathrm{C}$ in January and $36.75^{\circ} \mathrm{C}$ in July. The study area is in an arid bioclimatic stage with cool winters.

This orchard, of one hectare of surface, was installed in 1992 and includes a total of about 100 trees of apricot variety of Louizi or "Louzired" . The trees are cultivated in semi-intensive form with $6 \mathrm{~m}$ x $6 \mathrm{~m}$ spacing; each tree measures $3 \mathrm{~m}$ in height. Soil is a clay-silty texture and it is irrigated by a channel. In addition, fertilization of the soil with farm manure is carried out during the period from December to January, the weed control applied is mechanical in the cuvettes and the orchard does not undergo any phytosanitary treatment.

The study of the entomofauna was carried out between October 2014 to May 2015. We have adopted a qualitative sampling (classical hunting) (Colas, 1974) and a quantitative sampling using different methods: striking (Fauvel et al., 1981), trapping (colored traps, Barber pots, etc.) and mowing (Benkhelil, 1991).

Samples were kept at the laboratory in petri dishes or in labelled flasks. They were checked under the binocular microscope for the sorting and counting and they were determined using morphological characters. The determination was made using various identification keys: Perrier (1964), Chopard (1943), Severa (1988), Berland (1999), Dierl \& Ring (2009), Bouragba (2010), and Aguib (2014).

The indexes used to examine the bio-ecological status of the different insect groups in the apricot orchard during the study period (Magurran, 2004), were: Abundance frequency (FA) has been calculated for each order by the percentage of individuals in relation to the total $(\mathrm{N})$ recorded in the orchard, Species richness (S), Shannon's diversity index (H'), and Evenness (E).

\section{RESULTS AND CONCLUSIONS}

The results on the entomofauna sampled in the study area are shown on Table 1 with its numbers (ni) and its abundance frequencies (FA\%) depending on the species. All species are classified according to their taxonomic membership and diet (D).

The insect inventory has identified a total of 125 species belonging to 9 orders and 54 families with a total of 4,170 individuals (Table 1). Similar results were obtained by Naceri (2011) in an olive grove in Batna with a total of 156 species and 1528 specimens belonging to 16 orders and 80 families. Chafaa (2013) recorded in three olive groves in the region of Batna 206 species of insects with a total of 2311 specimens belonging to 11 orders and 74 families.

The species Henicopus sp., from the Dasytidae family (Coleoptera), is considered the most abundant species in our orchard with an abundance frequency of abundance of $42.45 \%$, followed by Cataglyphis bicolor of the Formicidae family (Hymenoptera) with a frequency of abundance of $5.18 \%$. Several authors show the dominance of Coleoptera in the region of Batna: Kellil (2010) in cereal fields, Zireg (2011) in a juniper forest, and Naceri (2011) in an olive grove. Guettala-Frah (2009) shows the dominance of Coleoptera (38\%) in an apple orchard in the Aurès region (Batna Department).

Among the species recorded, we have the presence of 4 species protected by regulations in Algeria (Decree No. 83-509 of 20/08/1983) related to nondomestic animal species. These species are: Coccinella septempunctata, Apis mellifera, Cataglyphis bicolor, and Polistes gallicus.

The diversity parameters calculated for the different types of the diet are recorded in Table 2. According to the results, we note a dominance of phytophagous with 71 species, followed by predators with 31 species and polyphagous with 13 species. We report a very low number of saprophagous (5), parasites (4) and coprophagous (1) species.

Phytophagous insects are very selective for plant species that they prefer. All parts of the plant can be attacked by xylophagous, frondicultural, radicicols, Saproxylic, and floricols insects (Villiers, 1979; Ricklefs \& Miller, 2005). 


\begin{tabular}{|c|c|c|c|c|}
\hline Families & Species & D & $\mathrm{Ni}$ & $F A(\%)$ \\
\hline \multicolumn{5}{|l|}{ Blattoptera } \\
\hline Blattidae & Blatta orientalis & Pol & 4 & 0.10 \\
\hline \multicolumn{5}{|l|}{ Coleoptera } \\
\hline Aphodiidae & Aphodius sp. & Cop & 1 & 0.02 \\
\hline Buprestidae & Acmaeoderella discoidea & Phy & 44 & 1.06 \\
\hline Buprestidae & Capnodis tenebrionis & Phy & 2 & 0.05 \\
\hline Buprestidae & Perotis unicolor & Phy & 1 & 0.02 \\
\hline Cantharidae & Cantharis sp. & Pre & 5 & 0.12 \\
\hline Cantharidae & Cantharis sp 1 & Pre & 4 & 0.10 \\
\hline Carabidae & Amara sp. 1 & Pre & 2 & 0.05 \\
\hline Carabidae & Amara sp. 2 & Pre & 4 & 0.10 \\
\hline Carabidae & Calathus fuscipes algiricus & Pre & 64 & 1.53 \\
\hline Carabidae & Calathus sp. & Pre & 1 & 0.02 \\
\hline Carabidae & Carabus sp. & Pre & 2 & 0.05 \\
\hline Carabidae & Lebia trimaculata & Pre & 1 & 0.02 \\
\hline Cetoniidae & Aethiessa floralis & Phy & 3 & 0.07 \\
\hline Cetoniidae & Cetonia funeraria & Phy & 4 & 0.10 \\
\hline Cetoniidae & Tropinota hirta & Phy & 12 & 0.29 \\
\hline Chrysomelidae & Chrysolina sp. & Phy & 3 & 0.07 \\
\hline Chrysomelidae & Cryptocephalus rugicollis & Phy & 1 & 0.02 \\
\hline Chrysomelidae & Entomoscelis rumicis & Phy & 6 & 0.14 \\
\hline Chrysomelidae & Oulema melanopus & Phy & 9 & 0.22 \\
\hline Cleridae & Trichodes alvearius & Phy & 6 & 0.14 \\
\hline Coccinellidae & Coccinella septempu & Pre & 4 & 0.10 \\
\hline Curculionidae & Hypera postica & Phy & 1 & 0.02 \\
\hline Curculionidae & Larinus sp. 1 & Phy & 1 & 0.02 \\
\hline Curculionidae & Larimus sp. 2 & Phy & 9 & 0.22 \\
\hline Curculionidae & Lixus sp. & Phy & 5 & 0.12 \\
\hline Curculionidae & Plagiographus excoriatus & Phy & 3 & 0.07 \\
\hline Curculionidae & Rhytidoderes plicatus & Phy & 2 & 0.05 \\
\hline Curculionidae & Scolytus rugulosus & Phy & 15 & 0.36 \\
\hline Dasytidae & Henicopus sp. & Phy & 1770 & 42.45 \\
\hline Geotrupidae & Geotrupes sp. & Sap & 3 & 0.07 \\
\hline Meloidae & Mylabris schreibersi & Phy & 1 & 0.02 \\
\hline Meloidae & Mylabris sp. & Phy & 1 & 0.02 \\
\hline Melolonthidae & Amphimallon sp. & Phy & 1 & 0.02 \\
\hline Melolonthidae & Rhizotrogus pallidipennis & Phy & 6 & 0.14 \\
\hline Melolonthidae & Rhizotrogus sp. & Phy & 7 & 0.17 \\
\hline Scarabaeidae & Scarabaeidae & Phy & 1 & 0.02 \\
\hline Staphylinidae & Othimus punctulatus & Pol & 2 & 0.05 \\
\hline Staphylinidae & Philonthus politus & Pol & 1 & 0.02 \\
\hline Staphylinidae & Staphylimus sp. & Pol & 9 & 0.22 \\
\hline Tenebrionidae & Adesmia faremonti & Pre & 1 & 0.02 \\
\hline Tenebrionidae & Omophlus sp. & Pre & 3 & 0.07 \\
\hline
\end{tabular}

\begin{tabular}{|c|c|c|c|c|}
\hline Families & Species & D & $\mathrm{Ni}$ & $\mathrm{FA}(\%)$ \\
\hline Tenebrionidae & Sepidium variegatum & Sap & 3 & 0.07 \\
\hline Tenebrionidae & Tentyria sp. & Sap & 1 & 0.02 \\
\hline Tenebrionidae & Tentyria thunbergi & Sap & 2 & 0.05 \\
\hline Tenebrionidae & Tribolium sp. & Sap & 2 & 0.05 \\
\hline \multicolumn{5}{|l|}{ Dermaptera } \\
\hline Forficulidae & Forficula auricularia & Pol & 1 & 0.02 \\
\hline \multicolumn{5}{|l|}{ Diptera } \\
\hline Anthomyiidae & Delia radicum & Pre & 1 & 0.02 \\
\hline Asilidae & Asilus sp. & Pre & 1 & 0.02 \\
\hline Bombyliidae & Bombylius major & Pre & 19 & 0.46 \\
\hline Calliphoridae & Calliphora vomitoria & Pol & 1 & 0.02 \\
\hline Calliphoridae & Lucilia caesar & Pol & 11 & 0.26 \\
\hline Culicidae & Culex pipiens & Pol & 28 & 0.67 \\
\hline Muscidae & Musca domestica & Pol & 562 & 13.48 \\
\hline Sarcophagidae & Sarcophaga carnaria & Pre & 96 & 2.30 \\
\hline Syrphidae & Scaeva pyrastri & Pre & 1 & 0.02 \\
\hline Syrphidae & Syrphus sp. & Pre & 100 & 2.40 \\
\hline Tachinidae & Gymnosoma rotundatum & Pol & 1 & 0.02 \\
\hline Tachinidae & Phryxe vulgaris & Pol & 12 & 0.29 \\
\hline Tachinidae & Tachina fera & Pol & 4 & 0.10 \\
\hline Tachinidae & Tachinus sp. & Pol & 12 & 0.29 \\
\hline \multicolumn{5}{|l|}{ Hemiptera } \\
\hline Anthocoridae & Anthocoris sp. & Phy & 80 & 1.92 \\
\hline Cicadellidae & Iassus lanio & Phy & 38 & 0.91 \\
\hline Cicadidae & Cicada sp. & Phy & 3 & 0.07 \\
\hline Cicadidae & Cicadetta sp. & Phy & 4 & 0.10 \\
\hline Pentatomidae & Pentatomidae sp. & Phy & 1 & 0.02 \\
\hline Reduviidae & Reduvius personatus & Phy & 1 & 0.02 \\
\hline Rhopalidae & Corizus cf. hyoscyami & Phy & 1 & 0.02 \\
\hline Scutelleridae & Eurygaster maura & Phy & 1 & 0.02 \\
\hline \multicolumn{5}{|l|}{ Homoptera } \\
\hline Aphididae & Macrosiphum rosa & Phy & 2 & 0.05 \\
\hline Aphididae & Myzus persicae & Phy & 1 & 0.02 \\
\hline Aphididae & Myzus sp. & Phy & 5 & 0.12 \\
\hline \multicolumn{5}{|l|}{ Hymenoptera } \\
\hline Apidae & Apis mellifera & Phy & 272 & 6.52 \\
\hline Apidae & Bombus sp. & Phy & 87 & 2.09 \\
\hline Apidae & Eucera punctatissima & Phy & 3 & 0.07 \\
\hline Apidae & Nomada cinnabarina & Phy & 1 & 0.02 \\
\hline Megachilidae & Rhodanthidium siculum & Phy & 5 & 0.12 \\
\hline Crabronidae & Larra anathema & Pre & 1 & 0.02 \\
\hline Chrysididae & Chrysis cuprea & Par & 8 & 0.19 \\
\hline Chrysididae & Chrysis ignita & Par & 3 & 0.07 \\
\hline Chrysididae & Chrysis trimaculata & Par & 2 & 0.05 \\
\hline
\end{tabular}

Table 1/1. Systematic list with inventoried insects, diet (D), numbers (ni) and frequency of abundance calculated for the study area (FA\%). Pol: Polyphaga, Phy: Polyphaga, Pre: Predators, Par: Parasites, Sap: Saprophagous, Cop: Coprophagous. 


\begin{tabular}{|c|c|c|c|c|}
\hline Families & Species & D & $\mathrm{Ni}$ & $\mathrm{FA}(\%)$ \\
\hline Chrysididae & Chrysis sp. & Par & 1 & 0.02 \\
\hline Formicidae & Aphaenogaster sp. & Pre & 15 & 0.36 \\
\hline Formicidae & Cataglyphis bicolor & Pre & 216 & 5.18 \\
\hline Formicidae & Cataglyphis sp. & Pre & 46 & 1.10 \\
\hline Formicidae & Crematogaster auberti & Pre & 56 & 1.34 \\
\hline Formicidae & Crematogaster scutellaris & Pre & 9 & 0.22 \\
\hline Formicidae & Crematogaster sp. & Pre & 1 & 0.02 \\
\hline Formicidae & Messor barbarus & Pre & 148 & 3.55 \\
\hline Formicidae & Tapinoma nigerrimum & Pre & 35 & 0.84 \\
\hline Halictidae & Sphecodes fuxipennis & Phy & 1 & 0.02 \\
\hline Megachilidae & Anthidium laterale & Phy & 1 & 0.02 \\
\hline Megachilidae & Hoplitis anthocopoides & Phy & 2 & 0.05 \\
\hline Megachilidae & Hoplitis sp. & Phy & 54 & 1.29 \\
\hline Megachilidae & Osmia anceps & Phy & 2 & 0.05 \\
\hline Megachilidae & Osmia pinguis & Phy & 6 & 0.14 \\
\hline Megachilidae & Osmia sp. & Phy & 60 & 1.44 \\
\hline Megachilidae & Osmia tricornis & Phy & 14 & 0.34 \\
\hline Megachilidae & Osmia tridentata & Phy & 2 & 0.05 \\
\hline Pompilidae & Anoplius sp. & Phy & 2 & 0.05 \\
\hline Sphecidae & Ammophila sabulosa & Pre & 1 & 0.02 \\
\hline Vespidae & Polistes gallicus & Pre & 1 & 0.02 \\
\hline Vespidae & Vespidae & Pre & 2 & 0.05 \\
\hline Vespidae & Vespula germanica & Pre & 1 & 0.02 \\
\hline \multicolumn{5}{|l|}{ Lepidoptera } \\
\hline Lycaenidae & Lycaena sp. & Phy & 29 & 0.70 \\
\hline Lycaenidae & Lycaena sp. & Phy & 2 & 0.05 \\
\hline Lycaenidae & Polyommatus icarus & Phy & 1 & 0.02 \\
\hline Nymphalidae & Melanargia sp. & Phy & 1 & 0.02 \\
\hline Nymphalidae & Vanessa cardui & Phy & 4 & 0.10 \\
\hline Papilionidae & Papilio machaon & Phy & 1 & 0.02 \\
\hline Pieridae & Colias croceus & Phy & 3 & 0.07 \\
\hline Pieridae & Pieris brassicae & Phy & 1 & 0.02 \\
\hline \begin{tabular}{|l|} 
Pieridae \\
\end{tabular} & Pieris rapae & Phy & 1 & 0.02 \\
\hline Pieridae & Pontia daplidice & Phy & 10 & 0.24 \\
\hline \multicolumn{5}{|l|}{ Orthoptera } \\
\hline \begin{tabular}{|l} 
Acrididae \\
\end{tabular} & Acrotylus patruelis & Phy & 1 & 0.02 \\
\hline \begin{tabular}{|l} 
Acrididae \\
\end{tabular} & Aiolopus thalassinus & Phy & 9 & 0.22 \\
\hline \begin{tabular}{|l|} 
Acrididae \\
\end{tabular} & Calliptamus italicus & Phy & 1 & 0.02 \\
\hline Acrididae & Sphingonotus sp. & Phy & 1 & 0.02 \\
\hline Gryllidae & Gryllus campestris & Phy & 3 & 0.07 \\
\hline Gryllidae & Gryllus sp. & Phy & 8 & 0.19 \\
\hline Gryllidae & Oecanthus pellucens & Phy & 7 & 0.17 \\
\hline Mantidae & Mantis religiosa & Pre & 1 & 0.02 \\
\hline \begin{tabular}{|l|} 
Pamphagidae \\
\end{tabular} & Ocneridia longicornis & Phy & 1 & 0.02 \\
\hline Pamphagidae & Ocneridia sp. & Phy & 3 & 0.07 \\
\hline Pyrgomorphidae & Pyrgomorpha cognata & Phy & 4 & 0.10 \\
\hline
\end{tabular}

Predatory species and polyphagous species are fairly well represented in study stations. Following Dajoz (2003), polyphagous species have a more eclectic diet. The polyphagous feed on animal and plant organic matter in different forms and they can therefore play a dual role. Predatory species can feed different prey during their life and their voracity is a useful indicator of their repressive potential; these characteristics affect the dynamics of predator-prey numerical interactions and the repressive effect expected from the study of predators (Hassell, 1978). A polyphagous predator uses several species of prey and the importance of each varies according to its relative availability (Coutier \& Cloutier, 1992). Coprophages, saprophagous and parasites are poorly represented in our inventory despite their roles in the functioning of agrosystems. Coprophages contribute to soil formation through burrowing and the incorporation of organic matter (Bachelier, 1978) and they help to structure the soil with the recycling of dung in the soil supplying nitrogen in the soil (Dajoz, 1985).

The saprophagous use all the dead substances, in particular the decaying vegetable detritus, by the action of the microorganisms, the mushrooms, and then the insects which will constitute the humus (Villiers, 1979; Lamy, 1999).

According to Beaumont \& Cassier (1983), there is no absolute trophic specialization in nature, so the distribution considers the type of diet of the adult states.

The Shannon index (H') for the three categories of phytophagous, predators and polyphagous is high compared to the other categories (parasites, saprophagous and coprophages) for the Ouled $\mathrm{Si}$ Slimane station (Table 2).

This index gives an idea of the diversity of all population considering not only the number of species, but also the number of individuals from the different trophic populations. A community is even more diverse when the Shannon's diversity index $\left(\mathrm{H}^{\prime}\right)$ is bigger. This index shows that the station Ouled Slimane is home to more diverse populations.

The equitability (E) of predators, polyphagous

Table 1/2. Systematic list with inventoried insects, diet (D), numbers (ni) and frequency of abundance calculated for the study area (FA\%). Pol: Polyphaga, Phy: Polyphaga, Pre: Predators, Par: Parasites, Sap: Saprophagous, Cop: Coprophagous. andophagous is high compared to parasites, coprophages and saprophagous (Table 2). This shows that the trophic categories in the study orchard are less balanced. 


\begin{tabular}{|c|c|c|c|c|c|c|}
\hline Coprophagous & Saprophagous & Parasites & Polyphagous & Predators & Phytophagous & \\
\hline 1 & 11 & 14 & 648 & 842 & 2654 & N \\
\hline 1 & 5 & 4 & 13 & 31 & 71 & S \\
\hline 0 & 0.02 & 0.03 & 0.42 & 0.47 & 0.41 & H' $^{\prime}$ \\
\hline 0 & 2.32 & 2 & 3.7 & 4.95 & 6.15 & H'max \\
\hline 0 & 0.01 & 0.01 & 0.11 & 0.09 & 0.07 & E \\
\hline
\end{tabular}

Table 2. Total richness (S), the Shannon index (H') and the equitability (E) of the insects identified by the diet.

\section{REFERENCES}

Achouri A., 1991. Evolution des dépérissements en fonction de l'âge et du stade végétatif de quelques vergers d'Abricotier dans la région de N'Gaous. Mémoire ingénieur. Université de Batna, Batna, Algérie, 71pp.

Aguib S., 2014. Biogéographie et Monographie des Megachilidae (Hymenoptera: Apoidea) dans le Nord Est Algérienne. Thèse doctorat. Université de Constantine, Constantine, $250 \mathrm{pp}$.

Bachelier G., 1978. La faune des sols: son écologie et son action. Ed. Office de la Recherche Scientifique et Technique Outre-Mer (O.R.S.T.O.M)., Paris, France, $391 \mathrm{pp}$.

Beaumont A. \& Cassier P., 1983. Biologie animale des protozoaires aux Métazoaires épithélioneuriens. Tom II. Ed. Dumon, Paris, 954 pp.

Benabbes R., 1990. Approche Nutritionnelle du dépérissement de l'Abricotier (Prunus armeniaca) dans la région de N'Gaous. Mémoire ingénieur. Université de Batna, Batna, 66 pp.

Benkhelil M., 1991. Les techniques de récolte et de piégeage utilisées en entomologie terrestre. Ed. Office Public University, Alger, 68 pp.

Berland L., 1999. Atlas des Hyménoptères de France Belgique et Suisse. Tome I. Ed. Boubée, Paris, 157 pp.

Bonsignore C.P. , Manti F. \& Vacante V., 2008. Field and tree distribution of Capnodis tenebrionis (Linnaeus, 1767) (Col. Buprestidae) adults in an apricot orchard in Italy. Journal of Applied Entomology 132: 216-224. https://doi.org/10.1111/j.1439-0418.2007. 01235.x

Bouragba B., 2010. Guides de Quelques Arthropodes en région Semi-aride. Ed .Institut National de Recherche Forestière, (INRF) Djelfa, $87 \mathrm{pp}$.

Bouzidi N., 1990. Inventaire de l'arthropodofaune phytophage et auxiliaire de l'Abricotier (Prunus armeniaca L.) et estimation des dégâts occasionnés par certains ravageurs dans la région de Ain Touta. Mémoire ingénieur. Université de Batna, Batna, 67 pp.
Bretaudeau J., 1979. Atlas d'arboriculture fruitière, Vol III. Ed. J.B. Bailliere, Paris, 184 pp.

ChafaaS., 1992. Contribution à l'étude Bio-écologique de la zoocoenose de trois vergers d'Abricotiers dans la région d'ouled Si Slimane (N'Gouas) Batna. Mémoire ingénieur, Institut Agro. Batna. 99 p.

Chafaa S., 2013. Contribution à l'étude de l'entomofaune de l'olivier. Olea europaea et de la dynamique des populations de la cochenille violette Parlatoria oleae Colvée, 1880 (Homoptera: Diaspididae) dans la région de Batna. Thèse Doctorat: Ecole National Supérieure Agronomique. El-Harrach. Alger, Algérie, $168 \mathrm{pp}$.

Chaouia C., 1987. Etude de comportement de quelques variétés d'Abricotier cultivées dans la station expérimentale de Boufarik (INAF). Mémoire ingénieur. INA. El-Harrach, Alger, 80 pp.

Chopard L., 1943. Faune de l'empire Français (Orthoptéroides de l'Afrique du Nord). Tome I. Ed. La Rose, Paris, 450 pp.

Cloutier C. \& Cloutier C., 1992. Les solutions biologiques de lutte pour la répression des insectes et acariens ravageurs des cultures. In: Vincent $\mathrm{Ch}$. \& Coderre D. (Eds). La lutte biologique. Ed. Lavoisier Tec \& Doc, Québec, 19-87 pp.

Colas G., 1974. Guide de l'entomologiste. Ed Boubée, Paris, 70 pp.

Dajoz R., 1985. Précis d'écologie, 5 ème édition. Ed. Dunod, Paris, 505 pp.

Dajoz R., 2003. Les coléoptères Carabidés et Ténébrionidés: Ecologie et Biologie. Ed. Lavoisier, Tec et Doc, Paris, $522 \mathrm{pp}$.

Derias M., 1984. Contribution à l'étude du dépérissement de l'Abricotier (Prunus armeniaca) dans la région de N'Gaous. Mémoire ingénieur. Institut national agronomique (INA), El-Harrach, Alger, 81 pp.

Dér Zs., Pénzes B. \& Orosz A., 2003. The Leafhopper Fauna of an Apricot Orchard in Hungary. Acta Phytopathologica et Entomologica Hungarica, 38: 145155. https://doi.org/10. 1556/APhyt.38.2003.1-2.17 
Dierl W. \& Ring W., 1992. Guide des insectes. Ed. Delachaux \& Niestlé. Paris. 237 pp.

Fauvel G., Rambier A. \& Balduque-Martin R., 1981. La technique du battage pour la surveillance des ravageurs en cultures fruitières et florales. Agronomie, 1: 105-113.

Guettala-Frah N., 2009. Entomofaune. Impact Economique et Bio-Ecologie des Principaux Ravageurs du Pommier dans la région des Aurès. Thèse doctorat. Université de Batna, Batna, 166 pp.

Hariri Gh., 1978. Les insectes économiques. Ed. Aoufest, Alep, 138 pp.

Ivanova L., 1991. Faunal investigations in the apricoat orchards in Bulgaria. Acta Horticulturae, 293: 533536. https://doi.org/10.17660/ActaHortic.1991.293.65

Ivanova L., 2006. Key-pests of apricoat and control. Acta Horticulturae, 701, 651-654. https://doi.org/ 10.17660/ActaHortic.2006.701.115

Kellil H., 2010. Contribution à l'étude du complexe entomologique des céréales dans la région des hautes plaines de l'Est algérien. Thèse magister. Université de Batna, Batna, 188 pp.

Lamy M., 1999. Les insectes et les hommes. Ed. Albin Michel. Paris, 96 pp.
Magurran A.E., 2004. Measuring biological diversity. Ed. Wiley-Blackwell, $256 \mathrm{pp}$.

Malek E., 1987. Les insectes des rosacées. Thèse d'université Damas. Damas, 106 pp.

Naceri T., 2011. Contribution a l'étude de l'arthropodohaune de l'Olivier dans une oliveraie à Boumia (W. de Batna). Mémoire Ingénieur. Université de Batna, Batna, 78 pp.

Perrier R., 1964. La faune de la France, Tome VI: Les Coléoptères 1ère Partie. Ed. Lib. Delagrave, Paris, $192 \mathrm{pp}$.

Ricklefs R.E. \& Miller G.L., 2005. Ecologie. Ed. De Boeck \& Larcier. Bruxelles, 821 pp.

Severa Z., 1988. Guide des insectes. Ed. Hatie, Prague, $318 \mathrm{pp}$.

Vidaud J., 1980. L'Abricotier. Ed. Centre technique interprofessionnel des fruits et légumes. Paris, $219 \mathrm{pp}$.

Villiers A., 1979. Initiation à l'entomologie. Anatomie. Biologie et Classification. Ed. Boubée \& Cie, Paris, $324 \mathrm{pp}$.

Zireg S., 2011. Diagnostique écologique, mise en valeur et conservation des juniperaies de Juniperus phoenicea de la région de Djerma (Nord-est de parc national de Belezma). Mémoire de Magistère. Université de Batna, Batna, Algérie, 104 pp. 